\title{
THE COMPLEX NATURE OF THE SEYFERT GALAXY NGC 7592
}

\author{
Piero Rafanelli \\ Department of Astronomy, University of Padova, Italy \\ Paolo Marziani \\ International School for Advanced Studies, Trieste, Italy
}

\section{Morphology}

NGC $7592 \equiv$ VV $731 \equiv$ MCG-01-59-017 is a system of close interacting galaxies. Two main galactic bodies are present in the CCD R-band image taken at the $1.8 \mathrm{~m}, \mathrm{~F} / 9$ telescope of the Asiago Observatory and shown in Figure 1:

- a north-western (NW) component ( $\equiv \mathrm{VV}$ 731B), whose starlike nucleus (A) shows a Seyfert-type spectrum (Archipova et al., 1981). A is surrounded by a nearly spheroidal envelope, with an extension in the west at $P . A .=290^{\circ}$ detected up to a distance from $\mathrm{A}$ of $\approx 9$ arcsec, which corresponds to $\approx 2.5 h^{-1} \mathrm{kpc}$ of projected linear distance $\left(1\right.$ arcsec $\approx .35 \mathrm{~h}^{-1} \mathrm{kpc}$ at the redshift $\left.z=0.0244 ; H_{0}=100 \mathrm{~h} \mathrm{~km} \mathrm{s^{-1 }} \mathrm{Mpc}^{-1}\right)$. This extension seems to bend in the northern direction and to join a bright wing, detected up to $\approx 22$ arcsec from $A$, which turns to east and resembles a spiral arm or a tidal tail.

- The envelope around $A$ is connected (over $\sim 20$ arcsec $\approx 7 h^{-1} k p c$ ) to a second galactic body (SE) located in the south-east of it. Its nucleus (B), identified by Markarian and Lipovetskii (1976) as the nucleus of Mkn 928, is located at $d_{A B} \sim 11 \operatorname{arcsec}\left(\sim 4 h^{-1} k p c\right)$ from $A$ at $P . A .=100^{\circ}$. The morphology of this component is highly peculiar. In the central region, $B$ is linked to two fainter knots which extend up to $d^{\prime \prime} \sim 4$ arcsec at $P . A .=90^{\circ}$. This structure gives to the region surrounding $\mathrm{B}$ an elongated and distorted shape. Moreover, a bar-like structure is detected on either sides of $B$. The north-eastern side of the bar $\left(P . A .=40^{\circ}\right)$ joins a slightly distorted loop of condensations, which can be traced from $P . A .=335^{\circ}$ (at a distance from $\mathrm{B} \approx 6$ arcsec $\approx 2.1 \mathrm{~h}^{-1} \mathrm{kpc}$ ) to $P . A .=100^{\circ}$ and from $P . A .=200^{\circ}$ to $P . A .=240^{\circ}$, but not in corrispondence of the contact region between the two galaxies. This structure seems to be drained and distorted in the direction of a third condensation $C$ at $d_{B C} \approx 11.7 \operatorname{arcsec}\left(\approx 4.1 h^{-1} \mathrm{kpc}\right)$ from $\mathrm{B}$ at $P . A .=212^{\circ}$. A faint plume bent in a direction opposite to that expected for spiral arms of the SE galaxy appears to extend from the west side of $\mathrm{C}$.

\section{Spectroscopy}

Long slit spectra of NGC 7592 were taken on Sep. 26-30, 1989 at the $1.52 \mathrm{~cm}$ ESO telescope, equipped with a Boller and Chivens spectrograph and an RCA High Resolution CCD camera. The covered spectral ranges are $3700-7300 \AA$ with resolution $\approx 3.7 \AA$, and $4500-5500 \AA$ and $6300-7300 \AA$ with resolution $\approx 1.8 \AA$. NGC 7592 was observed at two position angles: P.A. $=212^{\circ}$ (along $\mathrm{B}$ and $\mathrm{C}$ ), and $P . A .=100^{\circ}$ (along $\mathrm{B}$ and $\mathrm{A}$ ), with a slit width of $\approx 2$ arcsec $\approx 700 h^{-1} p c$.

\subsection{Physical conditions}

The $\mathrm{H} \alpha, \mathrm{H} \beta$, [OIII] $\lambda \lambda 4959,5007,[\mathrm{NII}] \lambda \lambda 6548,6583$, [SII] $\lambda \lambda 6717,6731$, and [OII] $\lambda 3727$ emission lines have been found to be spatially extended. The strongest ones, $\mathrm{H} \alpha$ and $[$ OII $] \lambda 3727$, are detected over $\approx 30$ arcsec $\approx 10.5 h^{-1} \mathrm{kpc}$ at $P . A .=100^{\circ}$ and $\approx 28$ arcsec $\approx$ $9.8 h^{-1} k p c$. The size of the emitting regions is comparable with the size of the galaxies belonging to the system NGC 7592 measured on its broad band image. A more refined analysis of the cross dispersion profile of $\mathrm{H} \alpha$ permits to isolate 6 different emitting regions 


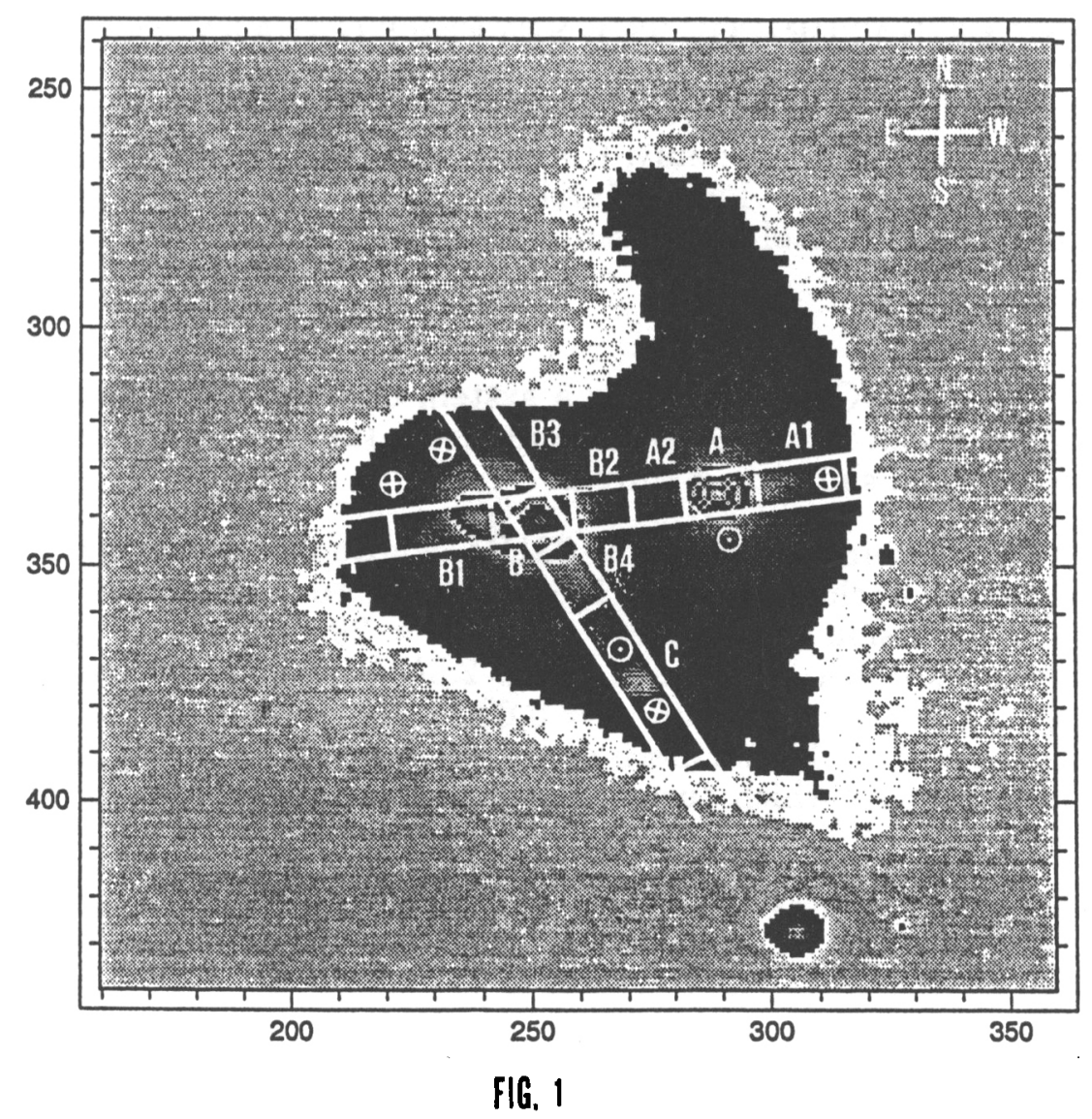

(A1,A,A2,B2,B,B1) along P.A. $=100^{\circ}$ and 4 regions $(\mathrm{C}, \mathrm{B} 4, \mathrm{~B}, \mathrm{~B} 3)$ 'along $P . A .=212^{\circ}$. The slit positions and the regions analyzed are marked in Figure 1.

The diagnostic diagrams proposed by Veilleux and Osterbrock (1987) were used to analyze whether the ionization of the line emitting gas is produced by a thermal (HII regions) or by a non-thermal continuum (AGN), Figures $2 a, 2 b, 2 c$. The Seyfert nucleus A of the NW component and A2, a region very close to $\mathrm{A}$ on its eastern side, are photoionized by a non-thermal source. All the other emitting regions are in the area typical of HII regions. This finding has been also tested using the diagnostic diagram based on the line ratios [OII] $\lambda 3727 /[\mathrm{OIII}] \lambda 5007$ and [OIII] $\lambda 5007 / \mathrm{H} \beta$ (Baldwin et al., 1981), which further allows to distinguish between gas photoionized by thermal sources and shock-heated gas. We found that A and A2 are the only regions not located in the HII area.

The spectrum of the Seyfert nucleus A shows a faint broad component of $\mathrm{H} \alpha$, already suspected by Archipova et al. (1981), whose FWZI measured in the high resolution spectra is $3200 \mathrm{~km} / \mathrm{s}$ (Figure 3a). An upper limit to its flux is $5 \times 10^{-14} \mathrm{erg} \mathrm{cm}^{-2} \mathrm{~s}^{-1}$, being $H \alpha_{B C} / H \alpha_{N C} \approx 0.7$. Since no broad component is present in $\mathrm{H} \beta, \mathrm{A}$ can be classified as a Seyfert 1.9 nucleus. The FWZI of the [OIII] $\lambda \lambda 4959,5007$ lines is $\sim 1500 \mathrm{~km} \mathrm{~s}^{-1}$, namely about twice the FWZI of $\mathrm{H} \beta, \sim 800 \mathrm{~km} \mathrm{~s}$. This is due to the presence of a semi-broad component of the $[\mathrm{OIII}] \lambda \lambda 4959,5007$ lines displaced toward the red (Figure $3 \mathrm{~b}$ ). The semibroad component indicates that there is a region whose density is larger than the density of the Narrow Line Region of $A, n_{e, A} \geq 2 \times 10^{2} \mathrm{~cm}^{-3}$ (estimated employing the [SII] $\lambda 6731 / \lambda 6716$ ratio, after correcting for the $\mathrm{B}$-band absorption) and smaller than the density typical of the Broad Line Region $n_{e}>10^{\circ} \mathrm{cm}^{-3}$. The active nucleus ionizes the interstellar gas between $A$ and $B$ (see also the results on the kinematics), and provides the main ionization source up to a distance of $5.1 \operatorname{arcsec} \approx 1.8 h^{-1} \mathrm{kpc}$ (region A2). It is noteworthy that outside of A and 

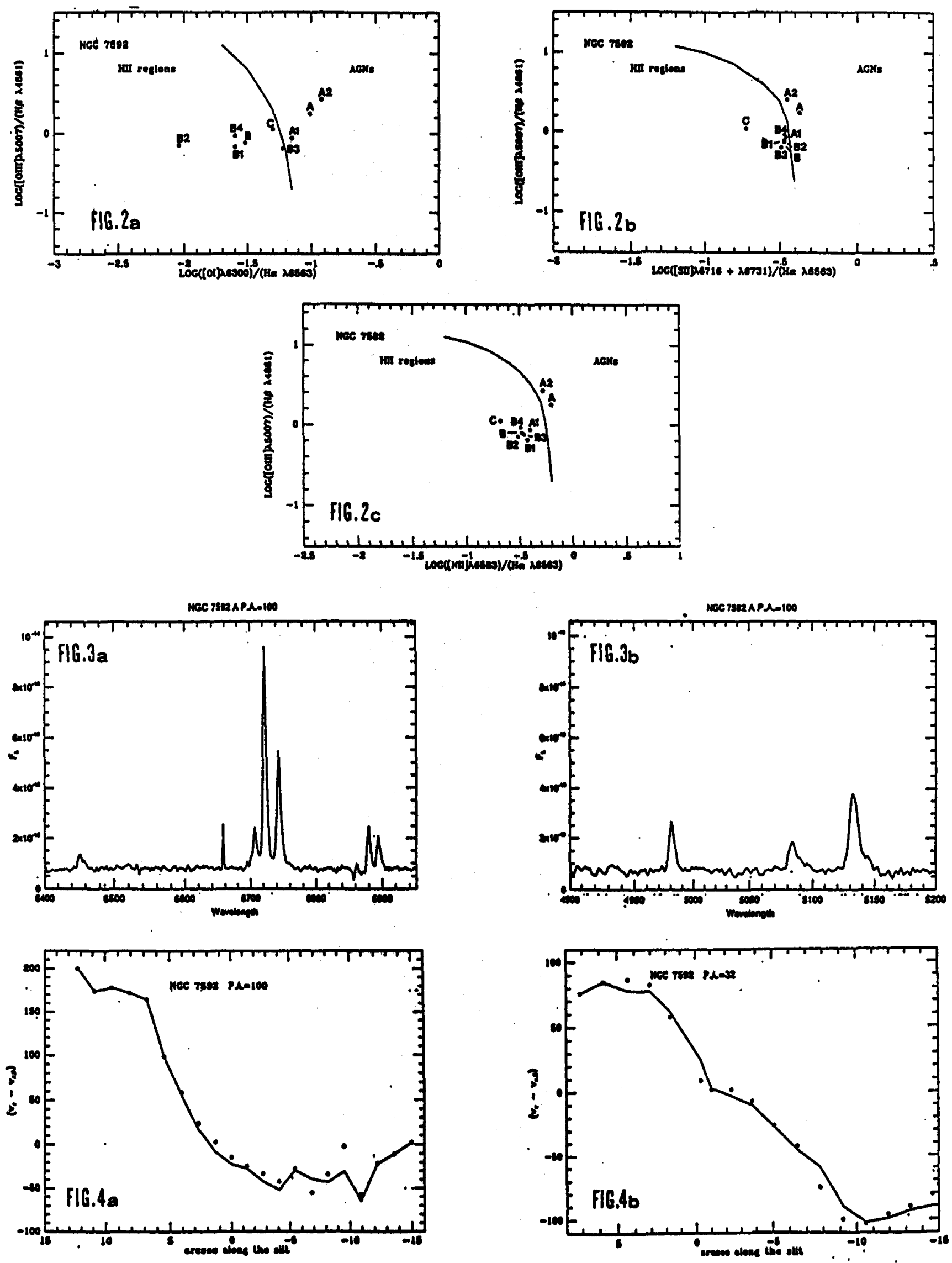
A2 the line emission is due to photoionization by a thermal continuum provided by $\mathrm{O}$ and $\mathrm{B}$ stars, and that the $\mathrm{H} \alpha$ luminosity of $\mathrm{A}, L(H \alpha)_{A} \approx 4.8 \times 10^{40} \mathrm{~h}^{-1} \mathrm{erg} \mathrm{s}^{-1}$ is less than that of $\mathrm{B}$ and $\mathrm{C}$, being $L(H \alpha)_{B} \approx 6.1 \times 10^{40} h^{-1} \mathrm{erg} \mathrm{s}^{-1}$ and $L(H \alpha)_{C} \approx 5.4 \times 10^{40} \mathrm{~h}^{-1} \mathrm{erg} \mathrm{s}^{-1}$. A comparison with the $\mathrm{H} \alpha$ luminosity function for starburst galactic nuclei published by Kennicutt et al. (1989) shows that the $\mathrm{H} \alpha$ luminosities of $\mathrm{B}$ and $\mathrm{C}$ are typical of this class of objects and that they are too high for disk HII regions. The $\mathrm{H} \alpha$ luminosities of the regions $\mathrm{A} 1, \mathrm{~B} 1, \mathrm{~B} 2, \mathrm{~B} 3, \mathrm{~B} 4$ range from $\approx 2 \times 10^{40} \mathrm{erg} \mathrm{s}^{-1}$ to $\approx 3 \times 10^{40} \mathrm{erg} \mathrm{s}^{-1}$ and are located on the high luminosity tail of the luminosity function of disk HII regions of Kennicutt et al.(1989).

\subsection{Kinematics}

The velocity curves at $P . A .=100^{\circ}$ and at $P . A .=212^{\circ}$ are shown in Figure $4 \mathrm{a}$ and $4 \mathrm{~b}$ respectively. The heliocentric radial velocity $\left(V_{r, B}=7320 \pm 10 \mathrm{~km} \mathrm{~s}^{-1}\right)$ and the position of $\mathrm{B}$ have been taken as zero points of the coordinates. The velocity curve at $P . A .=212^{\circ}$ is described by a straight line from $\approx-7$ arcsec to $\approx+3$ arcsec. This region corresponds to the bar-like structure revealed by the $R$ image. The velocity curve considerably flattens beyond both edges of the linear part, and a shallow decrease begins in corrispondence of $\mathrm{C}$ $\left(d^{\prime \prime} \sim 12\right.$ arcsec $)$, which has an heliocentric radial velocity $V_{r, C}=7230 \pm 10 \mathrm{~km} \mathrm{~s}^{-1}$. At P.A. $=100^{\circ}$, on the eastern side of $\mathrm{B}$ a steep velocity gradient is present up to $\approx 7$ arcsec, and it is followed by a flatter part, which can be traced up to $\approx 12$ arcsec. As a whole, the velocity curve resembles that of a normal spiral galaxy.

On the western side of $\mathrm{B}$, the gas motions are peculiar. A contour plot of the spectral region around $\mathrm{H} \beta$ and $\mathrm{H} \alpha$ (Figures $5 \mathrm{a}$ and $5 \mathrm{~b}$ ) shows that the gas turns to higher radial velocities $\left(v_{r}\right.$ increases from $\approx 7300 \mathrm{~km} \mathrm{~s}^{-1}$ to $\left.\approx 7490 \mathrm{~km} \mathrm{~s}^{-1}\right)$ up to the position of A. In correspondence of $A$ the profiles of the lines $\mathrm{H} \alpha, \mathrm{H} \beta$ and the [NII] $\lambda \lambda 6548,6583$ are double and show:

1. a strong blue component, at heliocentric radial velocity $v_{r, A}=7280 \mathrm{~km} \mathrm{~s}^{-1}$, which is due to the Narrow Line Region of the Seyfert nucleus. $v_{r, A}$ has been assumed as the systemic velocity of the Seyfert nucleus;

2. a fainter red component, related to the extended emitting regions connecting $\mathrm{B}$ with $\mathrm{A}$. Its peak velocity is $v_{r} \approx 7445 \mathrm{~km} \mathrm{~s}^{-1}$. This component may give a contribution up to $\approx 30 \%$ to the total $\mathrm{H} \alpha$ flux (the value given above may therefore be overestimated by the same fraction).

On the western side of $A(A 1)$ the velocity curve turns swiftly toward higher radial velocities.

\section{Discussion}

The problem of the nature of $\mathrm{C}$ is addressed at first. $\mathrm{C}$ shows an heliocentric radial velocity very similar to that of $A$ and $B$. Moreover, the arm departing from $C$ is most probably a tidal tail, because its extension is large and its orientation is peculiar. The high $\mathrm{H} \alpha$ luminosity of $\mathrm{C}$ is typical of a starburst nucleus. These facts argue in favour of $\mathrm{C}$ being the nucleus of a third galactic component (southern component S) physically interacting with the SE component of NGC 7592. The directions of the velocity vectors in various regions of NGC 7592 are marked in Figure 1. It is noteworthy that the SE component rotates clockwise, if the radial velocity difference $\Delta v_{r}$ from its nucleus $\mathrm{B}$ is due to rotation. Under the same assumption for the $\Delta v_{r}=v_{r}-v_{r, A}$, the NW component seems to rotate counterclockwise. Thus, the gas in the regions where the two galactic bodies are in contact moves in the same way, suggesting that a prograde encounter is occurring. It is known (e.g. Toomre and Toomre, 1972) that prograde encounters have the most disruptive effects on the interacting galaxies, leading to the formation of tidal tails. The interpretation of the wing of the $\mathrm{NW}$ component in terms of a tidal tail thus appears very likely. Figure 1 shows that a similar situation holds for the interaction between $\mathrm{SE}$ and $\mathrm{S}$ too, where $\mathrm{S}$ rotates counterclockwise. The interpretation of the arm departing from $\mathrm{C}$ as a tidal tail is supported also in this case. The difference in radial 
NGC 7592 P.A. $=100$

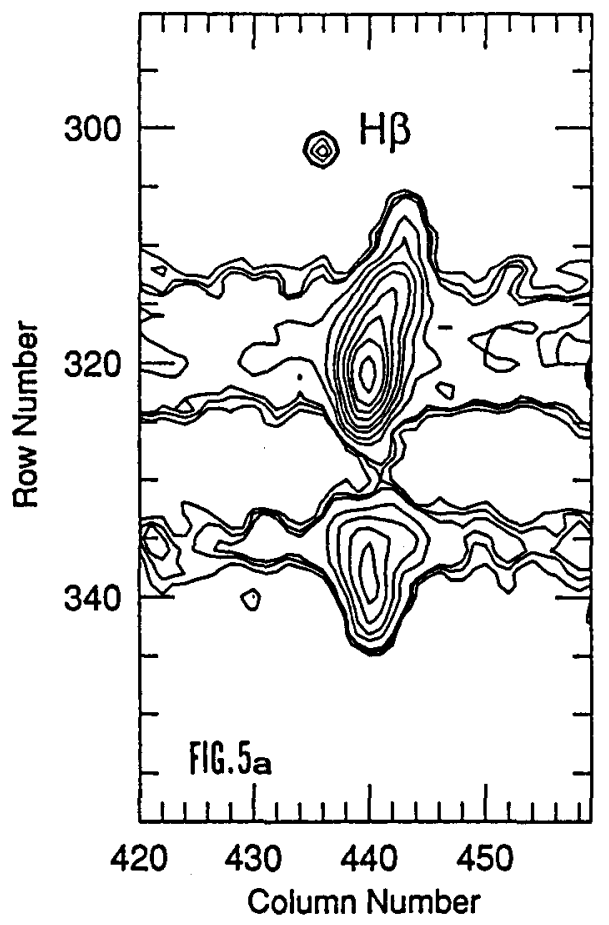

NGC 7592 P.A. $=100$

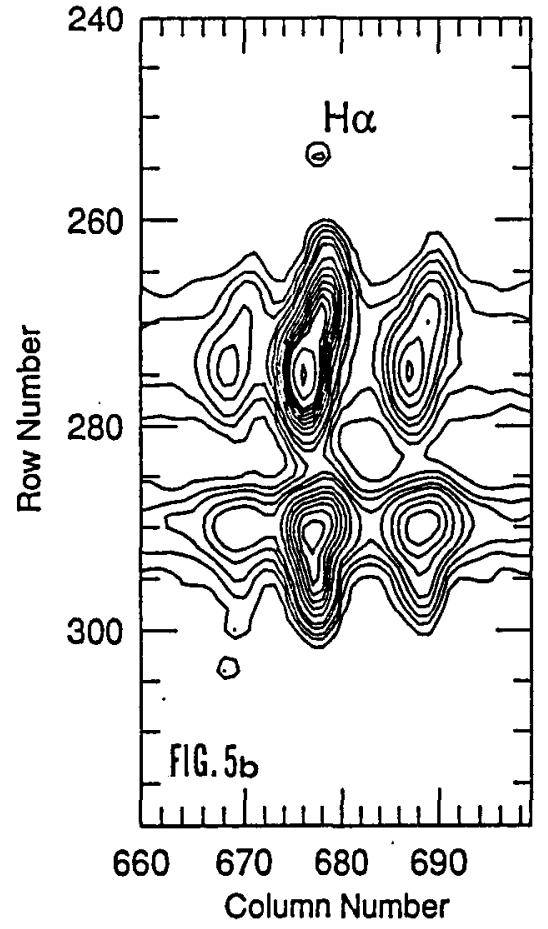

velocity between $A$ and $B\left(\Delta v_{r} \approx-40 \mathrm{~km} \mathrm{~s}^{-1}\right)$ and the morphology of NGC 7592 suggest that the NW component is beyond the SE one and is approaching it. The most heavily reddened regions $(E(B-V) \approx 0.7$, derived from the $\mathrm{H} \alpha / \mathrm{H} \beta$ ratio) are $\mathrm{B} 2$ and $\mathrm{B} 4$, namely the regions between $A$ and $B$. Moreover, the small difference in radial velocity between $A, B, C$ suggests that the three galaxies form a bound system, and their kinematics hints that they are most probably in the early stage of a merging phenomenon. In addition, the emitting gas connected to the red component of $\mathrm{H} \alpha$ on $\mathrm{A}$ appears to regress from the observer. An appealing hypothesis would be to consider the line emitting gas as infalling toward $A$, perhaps ultimately accreting onto the central core of the active nucleus.

\section{References}

Archipova V.P. et al.: 1981, Soviet Astron. 25, 277

Baldwin J.A., Phillips M.M., Terlevich R.: 1981, P.A.S.P. 93,5

Kennicutt R.C., Keel W.C., Blaha C.A.: 1989, Astron. J. 97, 1022

Markarian B.E, Lipovetskii V.A., Stefanian D.A. :1977, Astrofisica 13 , 225

Veilleux S., Osterbrock D.E.: 1987, Astroph. J. Suppl. 63, 295

Toomre A., Toomre J.: 1972, Astroph. J., 178, 623 